\title{
La sylviculture de haute qualité du chêne en Suisse : concepts d'éducation et de traitement dans des conditions écologiquement marginales
}

\author{
JP Schütz \\ Chaire de sylviculture, École polytechnique fédérale, CH 8092 Zürich, Suisse
}

(Reçu le 6 janvier 1993; accepté le 2 juin 1993)

\begin{abstract}
Résumé - La sylviculture du chêne de haute qualité en Suisse se différencie significativement en certains points de celle pratiquée dans les grandes régions traditionnelles de production, de France et d'Allemagne notamment. Cela tient aussi bien à des particularités stationnelles qu'à des priorités différentes des concepts de sylviculture. En Suisse, la culture du chêne de qualité se conçoit d'abord sur des stations de la hêtraie en l'absence pour des raisons climatiques (précipitations élevées) et édaphiques (sols jeunes, riches et bien tamponnés) de véritables chênaies permettant une production de valeur. De sucroît, le caractère généralement montagneux du pays et l'altitude relativement élevée des forêts (plus de 450 à $500 \mathrm{~m}$ ) ne permettent la culture du chêne que dans une zone à considérer comme marginale par rapport à la grande chênaie d'Europe centre-occidentale, car rapidement limitée par les gels tardifs et les dégâts de neige lourde. Outre ces 2 facteurs limitant la constitution des peuplements, les conditions d'alimentation hydrique et minérale très favorables conduisent à une productivité remarquablement élevée, de plusieurs classes de productivité plus élevée que dans les pays voisins. De telles stations conviennent mieux au chêne pédonculé qu'au rouvre. II y présente une croissance plutôt rapide. La sylviculture à pratiquer dans de telles conditions s'apparente plus à celle des forêts riveraines de type Slavonie qu'aux chênaies du Spessart ou du secteur ligérien français. Les principes d'une sylviculture de qualité, visant une proportion élevée de bois de très haute valeur $(>15 \%)$ doivent concilier des critères pas nécessairement concomitants, à savoir : des propriétés technologiques (finesse et régularité des bois) et de formation de la tige (critères sylvologiques). Alors que la sylviculture européenne du chêne est orientée principalement en fonction de critères de finesse des cernes, celle pratiquée en Suisse choisit de favoriser la qualité de la tige, en profitant d'un effet de sélection et conjointement en visant le développement d'un peuplement accessoire à fonction d'emballage. II s'agit d'une sylviculture active, qui ne réprime pas la croissance, mais au contraire profite de l'avantage économique d'une moindre durée de production, de 160 ans, pour optimiser les autres facteurs d'influence. Une telle sylviculture présente une parfaite cohérence entre les interventions sylvicoles et les objectifs de production. Les caractéristiques en sont de travailler sur une base génétique suffisante, permettant une sélection des meilieurs phénotypes, à caractère positif, encore modérée en prime jeunesse, devenant active au stade du perchis avec des interventions d'éclaircie sélective vigoureuses. On prend finalement position à propos des propositions récentes de régénérer le chêne par une conduite plus lente des
\end{abstract}


coupes et en travaillant par petites trouées. Dans les conditions de station présentées, de telles propositions apparaissent difficiles à réaliser.

éducation / traitement sylvicole / durée de production / peuplement accessoire / Quercus robur $=$ chêne pédonculé $/ Q$ petraea $=$ chêne rouvre

Summary - High-quality oak silviculture in Switzerland: concepts of education and production in the marginal range of European oak. High-quality oak silviculture in Switzerland differs significantly in some ways from traditional silviculture in classical oak-producing regions of centralwestern Europe such as France and Germany. Particularities regarding site conditions, concepts and priorities are the main reasons for these differences. Oak only occurs naturally on beech-forest sites in Switzerland. Natural oak formations are lacking because of the climatic (high annual precipitation) and edaphic conditions (pedogenetically young soils, which are well balanced and rich in minerals). Furthermore, due to the mountainous character of the land and the relative elevation of forest distribution even in the Swiss Midlands (> $450 \mathrm{~m}$ altitude), oak production is not only considered marginal compared to that in the classical oak producing regions of Europe but is also limited by frost damage and heavy snow. However, mineral content and water capacity of the soil are very favourable, leading to remarkable productivity and higher site indexes than in the classical oak-producing regions. Such site conditions are better suited to pedunculate ( $Q$ robur) than to sessile oak ( $Q$ petraea), which grows fairly rapidly under these conditions. Silvicultural concepts are therefore more closely associated with riparian pedunculate oak forests, eg, of Slavonia than to the sessile oak forests of Germany and France. The goal of high-quality oak silviculture in Switzerland is to produce a proportion of $\approx$ $15 \%$ veneer quality lumber. Different partially divergent aspects such as technological wood properties (regularity and width of the rings), bole formation and development or growth potential have to be taken into consideration to achieve this goal. Classical oak silviculture in Europe is mainly oriented to producing wood with regular narrow rings by maintaining high stand densities. Oak silviculture in Switzerland, however, seeks optimization of bole quality and high production via recurrent selection and simultaneously establishing or favouring an appropriate subordinate secondary stand which has a beneficial effect on bole quality. Diameter growth is not restricted by high competition as in classical oak silviculture; on the contrary, active silviculture tries to utilize the economic advantages of good diameter growth and a shorter production time of $\approx 160 \mathrm{yr}$, with good agreement between production goals and silvicultural measures. The success of this concept depends on a sufficiently broad genetic base to allow active and positive selection of the best phenotypes in the early stages, selection according to collective education at first and to individual education with vigorous selective thinning from the pole stage on onwards. Finally, a comment has been made regarding new tendencies in oak silviculture which call for a longer regeneration period, overlapping generations or regeneration in very small stand openings. Considering the previously mentioned site conditions and the intolerance of young oak to shade, especially pedunculate oak, these tendencies do not appear to be realistic.

education / silvicultural treatment / production period / secondary stand / Quercus robur = pedunculate oak $/ \mathrm{Q}$ petraea $=$ sessile oak

\section{PARTICULARITÉS STATIONNELLES DE LA SYLVICULTURE DES CHÊNES EN SUISSE}

Comparativement aux grandes régions traditionnelles de culture du chêne de prestige en Europe, la sylviculture de cette essence pratiquée en Suisse s'en distingue significativement à certains égards. Cela est dû en grande partie à des conditions de station particulières au Plateau suisse. Elles se caractérisent en effet par une pluviosité généralement assez élevée (plus de $1000 \mathrm{~mm} / \mathrm{an}$ ), un climat frais, tempéré, à caractère océanique et des sols jeunes, c'est-à-dire riches en particules fines et en éléments nutritifs. C'est ainsi que dans les 
formations forestières climaciques du Plateau suisse dominent essentiellement des hêtraies plus ou moins pures. Le chêne, à l'exception de stations pauvres et impropres à une production de bois de qualité, ne constitue pratiquement pas de massifs naturels. On le rencontre à l'état plus ou moins disséminé dans des massifs dominés par le hêtre et certains feuillus précieux tels que le frêne et l'érable sycomore. De surcroît la forêt suisse se trouve principalement située à des altitudes supérieures à 450 à $500 \mathrm{~m}$, c'est-à-dire à un niveau altitudinal rapidement limitatif au bon développement qualitatif des chênes, en raison d'une part des gels tardifs et surtout des dégâts de neiges lourdes. Ces 2 facteurs limitent en fait à $600-650 \mathrm{~m}$ d'altitude le domaine de réalisation d'une sylviculture de qualité, c'est-à-dire qui escompte réaliser une proportion d'assortiments de haut de gamme (notamment de bois de tranchage) d'au moins $15 \%$. C'est donc dans une bande altitudinale assez étroite, allant de $450 \mathrm{~m}$ à $650 \mathrm{~m}$ que se réalise la production de chênes de qualité en Suisse. Les concepts de production mis en place s'orientent en fonction des facteurs limitatifs prépondérants cités plus haut, et cela explique pourquoi notre conduite des peuplements de chênes se singularise notablement en certains points de la sylviculture classique dans les grandes régions traditionnelles de production en Europe centre-occidentale.

\section{LES PARTICULARITÉS DES CHÊNES PÉDONCULÉ, ROUVRE ET LEURS FORMES INTERMÉDIAIRES}

C'est le chêne pédonculé (Quercus robur L) qui convient mieux que le chêne rouvre (Quercus petraea [Matt] Liebl) aux conditions particulières de climat et de station précitées, même si les 2 espèces se rencontrent très intimement mêlées dans les forêts. Les bonnes réserves en eau des sols d'une part, mais surtout la meilleure résistance du chêne pédonculé aux gels tardifs d'autre part expliquent l'avantage indéniable de cette dernière essence. Les observations de la phénologie du débourrement effectuées par Burger (1921) dans les environs de Zurich ont démontré que les jeunes plants de chênes rouvres débourraient plusieurs jours avant ceux de chênes pédonculés. Des essais comparatifs réalisés à l'instigation de Burger, dans le premier tiers du siècle, en de nombreuses stations du Plateau suisse, corroborent cette observation. II s'avère que les chênes rouvres souffrent de dégâts de gel plus prononcés que les pédonculés (Burger, 1949), ce qui explique les différences de forme des tiges, significativement meilleures, c'est-à-dire moins sinueuses, chez les pédonculés que les rouvres, et cela sur toutes les stations testées (Burger, 1944). Ces différences restaient significatives plusieurs décennies plus tard (Schütz et Badoux, 1979).

À vrai dire, on ne rencontre dans les peuplements naturels pas seulement des chênes pédonculés et rouvres, mais toute une gamme de formes morphologiquement intermédiaires, produites au cours des générations par hybridations spontanées et croisements en retour introgressifs. Comme l'a montré Kissling (1983), la proportion et le niveau d'introgression de ce continuum d'hybrides se trouvent très étroitement liés aux conditions de station, notamment de sol. Sur les sols bien alimentés en eau se rencontrent des populations phénotypiquement assez pures de pédonculés, alors que les rouvres dont les formes dominent sur les stations plus sèches n'apparaissent pratiquement jamais sous une forme taxonomique pure, mais comprennent toujours une proportion plus ou moins élevée de caractères de pédonculé et d'ailleurs également de pubescent (Quercus pubescens Willd). Cela 
laisse entrevoir l'importance des conditions édaphiques, en particulier de l'alimentation hydrique sur les processus d'adaptation des espèces et races de chênes.

La grande hétérogénéité génétique au sein des populations natives et par voie de conséquence la grande diversité de formes issues de cet abâtardissement, ne constituent pas nécessairement un avantage sylvicole, notamment en ce qui concerne les possibilités de sélection. Les essais de provenances ont montré, en effet, que la proportion de tiges aux iormes médiocres est plus élevée chez les provenances helvétiques que celles des grandes régions étrangères de production (Schütz, 1979). C'est une des raisons pour lesquelles, au moment de la régénération des peuplements, on préfère généralement la plantation à la régénération naturelle, à plus fortes raisons parce que sur de telles stations, l'envahissement par la végétation adventice concurrentielle représente un sérieux handicap à une régénération naturelle. Dans l'optique d'une production de haute valeur, il convient alors d'utiliser des provenances de qualité, à introduire de préférence des régions de provenances reconnues de l'étranger.

\section{LA REMARQUABLE PRODUCTIVITÉ DES CHÊNES SUR LES STATIONS DE LA HÊTRAIE}

Sur les bonnes stations de hêtraie en Suisse, la productivité des chênes est remarquablement élevée. À 50 ans, la hauteur dominante (indice de fertilité) est en moyenne de $22 \mathrm{~m}$ (fig 1). Cela devrait correspondre à une hauteur dominante de plus de $30 \mathrm{~m}$ à 100 ans. Comparativement aux fertilités obtenues dans les régions de production de l'étranger (France, Alle-

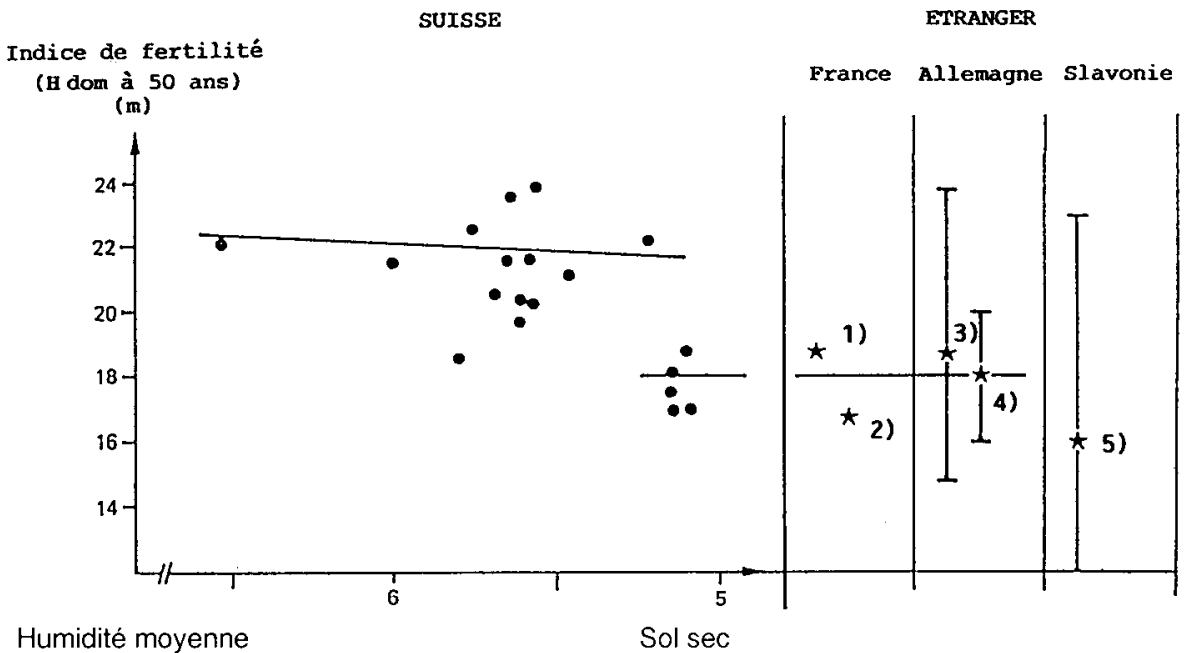

Fig 1. Indice de fertilité (Hdom à 50 ans) des parcelles d'essai de production de chênes pédonculés suisses en fonction des réserves hydriques des sols, et en regard des prestations selon les tables de production dans les chênaies étrangères (d'après Schütz, 1979). Données des placettes d'essai suisses (chênes pédonculés) individuelles. $\star$ Valeurs des tables de production : 1) d'après Lorne (1959) ; 2) d'après Pardé (1962) ; 3) d'après Mitscherlich (1953) ; 4) d'après Mayer (1960) ; 5) d'après Trifunovic (1965). En abscisse est portée la valeur indicatrice d'humidité du sol, donnée par le chiffre moyen des plantes des relevés floristiques selon la méthode de Ellenberg (1974). 
magne, Slavonie), les prestations en hauteur des chênes en Suisse sont de 2 à $4 \mathrm{~m}$ plus élevées, voire plus (Schütz, 1979). II est intéressant de constater que la croissance en hauteur des chênes pédonculé et rouvre ne se différencie pratiquement pas sur une même station. Le chêne s'avère être en jeunesse, c'est-à-dire dans la première moitié de la durée de production, une essence à bonne croissance qui dépasse indiscutablement en hauteur le hêtre et ne se trouve en reste que de peu par rapport à l'épicéa. La figure 1 illustre parfaitement la dépendance étroite de la hauteur des peuplements avec les conditions de station, notamment avec les réserves en eau utile du sol. En effet, pour les parcelles d'essai de production suisses, l'indice de fertilité (Hdom à 50 ans) ne se maintient à un niveau élevé que sur les stations aux réserves d'eau suffisantes, facteur représenté à la figure 1 par la valeur correspondante des plantes indicatrices des relevés de la végétation selon la méthode de Ellenberg (1974). Les remarquables prestations citées plus haut se réalisent sur la plupart des stations de la hêtraie. Sur celles à régime hydrique plus déficient, telles que dans le domaine des hêtraies à pulmonaires, on assiste à une chute très marquée de la productivité, qui se tient alors au niveau de celle des pays voisins. À de tels indices de fertilité correspondent des productivités en volume de 7 à $8 \mathrm{~m}^{3}$ d'accroissement moyen des chênes par ha et année.

En conclusion, nous avons affaire sur les bonnes stations du Plateau suisse à un chêne à croissance plutôt rapide, en mesure de fournir des arbres à fûts élevés, susceptibles de produire une proportion importante de bois d'œuvre en un laps de temps d'environ 160 ans et environ 80 à 100 tiges d'élite de dimensions excédant $65 \mathrm{~cm}$ de diamètre. II est vrai que cela ne se réalisera qu'avec la production de bois à cernes de 2 à $2,5 \mathrm{~mm}$ de largeur. Ces conditions se rapprochent plus de celles des chênaies de pédonculés des plaines alluviales, de type Slavonie, que de la chênaie de rouvre à croissance plus lente de la région ligérienne française, du Spessart ou du Palatinat allemand.

\section{PRINCIPES D'ÉDUCATION DES CHÊNAIES DE QUALITÉ}

Que l'objectif du traitement des chênaies de qualité consiste à obtenir une proportion la plus élevée possible d'assortiments de bois de haut de gamme, à savoir de tranchage, ne fait l'objet d'aucune divergence de vue chez les sylviculteurs. En revanche, quant aux moyens de réaliser ces objectifs, les avis sont partagés, parfois considérablement. Pour bien comprendre de tels points de vue apparemment contradictoires, il convient de s'interroger sur les différents facteurs permettant la réalisation de bois de qualité. Des possibilités d'influencer ces facteurs par des mesures sylvicoles appropriées dépendra la définition des concepts de traitement à appliquer.

Le premier critère nécessaire à l'obtention de bois de qualité, critère jusqu'à présent le plus souvent pris en considération en premier lieu, est celui de produire un bois à fine structure de cernes. À notre avis, on a donné une importance disproportionnée à ce critère par le passé, ce qui conduit à des concepts de production visant le maintien d'une densité de peuplement élevée, gage d'une croissance lente. Une telle vision repose, il est vrai, sur l'observation que les assortiments élitaires de chêne, aujourd'hui commercialisés, échoient à des billes à très fine structure de cernes. À vrai dire, ces observations ne s'avèrent pertinentes que pour les régions traditionnelles de production et seulement pour les assortiments supérieurs. Elles va- 
lent finalement essentiellement pour le chêne rouvre, qui dans ces régions croît de toutes façons relativement lentement. $\grave{A}$ l'opposé, on constatera également que dans les régions de plaines alluviales, telles que la Slavonie, on réalise des proportions élevées de bois de tranchage de qualité supérieure avec des bois de structure bien différente, à savoir des chênes pédonculés arrivant à terme de production à 140 ans (Trifunovic, 1965). II convient d'ajouter que la technique du tranchage a considérablement évolué, et permet aujourd'hui d'utiliser des bois à cernes de 2, voire $2,5 \mathrm{~mm}$ de large.

Un deuxième critère de réalisation de bois de qualité est d'ordre sylvologique, à savoir l'obtention d'arbres à fûts droits, réguliers et libres de branches de la plus grande longueur possible, qui de surcroît doivent rester libres de gourmands jusqu'au terme de la production. Cette condition repose sur la nécessité de pratiquer une sélection précoce selon la faculté de former une tige non partagée à bonne axialité et de bonne conformation des houppiers, ce qui implique des soins culturaux précoces (c'est-à-dire dès l'état de fourré) et des interventions éducatives vigoureuses dès le perchis. Finalement la réalisation de la condition selon laquelle les chênes de qualité doivent rester libres de gourmands jusqu'au terme de production est décisive dans le choix du concept de traitement. Elle ne peut se réaliser que grâce à un bon emballage des chênes d'élite par un peuplement accessoire éducatif, constitué d'essences commensales, restant si possible surbordonnées au chêne et n'exerçant qu'une faible concurrence sur les arbres de valeur; une vraie gageure! La question du peuplement accessoire revêt une importance majeure et n'est pas subsidiaire, comme on le concède parfois encore trop volontiers. En fin de compte, seul cet emballage vert des fûts est en mesure de contenir efficacement le développement des branches gourmandes.

C'est généralement à cette condition du bon développement du peuplement accessoire que s'achoppent les concepts de production qui escomptent contrôler le critère de largeur des cernes par le maintien de densité de peuplement élevée. Cette critique vaut aussi pour les concepts qui prévoient l'introduction d'un peuplement accessoire à la moitié de la durée de production. En effet, pour ce faire, il serait nécessaire de réduire significativement le couvert, avec pour conséquence le développement d'une couverture de gourmands, d'autant plus dense que les chênes ont été précédemment concurrencés et possèdent des houppiers étriqués. Jusqu'au moment où le peuplement accessoire ainsi favorisé devient fonctionnel, ces gourmands auraient plusieurs décennies pour se développer en véritables branches gourmandes dépréciant alors considérablement la qualité des tiges des chênes d'élite.

À l'inverse, l'idée de favoriser très rapidement le peuplement accessoire, c'est-àdire pratiquement dès la fin de l'état de fourrè, voire même en cas de création artificielle de peuplement dès la plantation, en intervenant par des mesures sylvicoles conséquentes et des interventions régulatrices, est un concept beaucoup plus cohérent. II permet de concilier les diverses interventions culturales, à savoir assurer un développement optimal du peuplement, pratiquer la sélection précoce et finalement favoriser les arbres d'élite choisis, en leur assurant un bon développement du houppier. En effet, comme nous le savons aujourd'hui, le bon encouronnement des arbres est une condition de contrôle du développement inopiné des gourmands tout aussi efficace que le maintien des fûts à l'ombre. Un houppier bien développé favorise la production d'hormones, qui maintiennent en dormance les bourgeons pro- 
ventifs et ainsi retiennent leur éclosion en gourmands (Roussel, 1977/1978).

\section{CONCEPTION SUISSE DE TRAITEMENT DES CHÊNES}

L'idée du traitement des chênes de qualité en interventions sylvicoles actives, dès le jeune âge s'est imposée naturellement en Suisse depuis longtemps, en parfaite concordance avec les conditions stationnelles décrites plus haut. Sans doute des facteurs tels que la médiocre qualité phénotypique des populations de chênes natifs et la nécessité d'assurer dès le plus jeune âge une stabilité suffisante contre les dégâts de neige lourde, ont contribué à démontrer les avantages d'interventions précoces. Depuis les travaux de Kodrik (1991) sur la sensibilité des jeunes peuplements de hêtre à l'égard de l'écrasement par les neiges lourdes, on sait que, aux altitudes critiques (500 à $600 \mathrm{~m}$ ) les arbres ne devraient pas excéder un coefficient d'élancement (rapport h:d) critique de 140. Ce critère étant difficile à percevoir à l'œil nu, il est préférable d'apprécier le degré d'encouronnement ou valeur relative de la longueur du houppier, qui ne devrait pas diminuer au-delà de 25 à $30 \%$. Ce qui vaut pour le hêtre vaut par analogie pour le chêne. De sucroît chez cette dernière essence, la faculté de conserver le feuillage marcescent, propre aux stades juvéniles, augmente considérablement la sensibilité aux dégâts de neige lourde. Quoi qu'il en soit, la conception suisse du traitement des chênes se fonde sur l'observation que le facteur "largeur de cernes» dépend plus de la station que du traitement et qu'il convient de considérer cette grandeur comme une donnée de départ sur laquelle le traitement sylvicole n'exerce qu'une influence modérée.

La figure 2 présente l'évolution entre 50 et 100 ans environ, de la largeur des cernes des éléments constitutifs du peuplement (c'est-à-dire des arbres à statut social dominant et codominant) de 4 parcelles d'essai sises sur différentes stations et traitées en éclaircies sélectives de force augmentant quelque peu avec l'âge. On constate, il est vrai, une légère augmentation de la largeur des cernes avec la diminution de la densité des peuplements consécutive aux éclaircies vigoureuses, mais cette influence apparaît relativement faible, moindre en tous cas que celle imprimée par la station et concrétisée par les différences de niveau des courbes. Des interventions d'éclaircies précoces présentent par ailleurs l'avantage incontestable de profiter au mieux de l'effet de sélection des arbres en fonction des propriétés de la tige. Elles permettent également de favoriser à temps le développement d'un peuplement accessoire consistant.

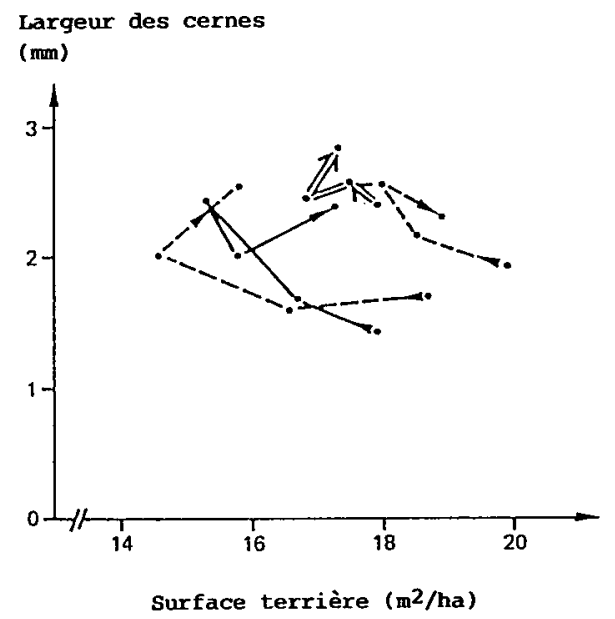

Fig 2. Largeur moyenne des cernes des arbres du peuplement principal (arbres dominants et codominants) de 4 parcelles d'essai de chênes pédonculés, entre 50 et 100 ans, en fonction de leurs surfaces terrières (d'après Schütz, 1979). Domaine stationnel (unités phytosociologiques): Aro-Fagetum ; - Milio-Fagetum; - Galio adorati Fagetum. 
Le critère déterminant de la sélection est le choix d'un nombre suffisant d'arbres d'élite selon leur faculté à former une tige continue, non partagée (axialité de la tige). Au terme de la production, le nombre d'arbres devrait se situer aux environs de 80 à 100 tiges/ha. Comme nous le savons depuis les travaux fondamentaux de Krahl-Urban (1953, 1959) on retrouve dans toute population de chênes, différents phénotypes, selon la faculté de dominance axiale. Ces différents types d'arbres (arbre fourchu, buissonneux et à bonne axialité) se reconnaissent très tôt (fig 3). Étant donné que chez une espèce comme le chêne, les positions sociales se figent assez tôt au cours du développement (Leibundgut, 1976), la conséquence en est que la sélection doit se faire également très tôt, c'est-à-dire déjà à l'état de fourré, avec des interventions actives et souvent répétées à rotation d'environ 5 ans, agissant dans la partie supérieure de la frondaison. Il s'agit au fourré d'intervenir de façon aussi bien négative que positive, avec pour objectif une homogénéisation qualitative et quantitative du massif (c'està-dire une intervention d'éducation collective) en visant un niveau de densité de

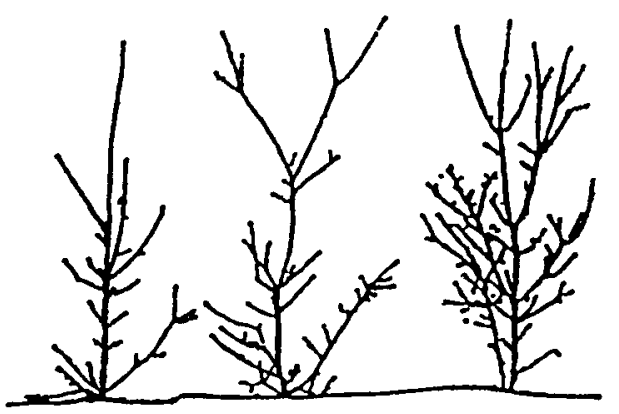

A

B

C

Fig 3. Les différentes formes de croissance des jeunes chênes (d'après Krahl-Urban, 1959). A : type à bonne axialité de la tige ; $C$ : type buisonneux ; $B$ : fourchu. peuplement variant selon les risques. La sélection se fait essentiellement selon le critère d'axialité de la tige, c'est-à-dire la faculté de former une tige continue, non subdivisée, à embranchement étalé. Étant donné que les chênes s'élaguent naturellement sans trop de difficultés (Butin et Kowalski, 1983), le facteur de la grosseur des branches n'est pas un critère décisif de sélection. La densité optimale des massifs, objectif important des opérations culturales, dépend donc moins du risque de formation de branches grossières que du risque d'écrasement par les neiges lourdes. La densité à retenir peut ainsi différer notablement selon les stations. Elle représente finalement un compromis entre d'une part la nécessité d'un entourage de congénères dans le collectif, favorable à une bonne éducation, et d'autre part la nécessité d'éclaircir le collectif pour en assurer la stabilité. On ajoutera que la bonne résistance à l'écrasement par les neiges lourdes dépend aussi de facteurs génétiques, à savoir la faculté des jeunes chênes à conserver ou non le feuillage marcescent en hiver, faculté qui peut varier notablement selon les provenances.

Les réflexions concernant le peuplement accesșoire interviennent dès la création du peuplement principal. Si ce dernier est créé par voie artificielle, il convient d'implanter de préférence le peuplement accessoire en même temps que l'essence principale. On utilisera alors des espèces tolérantes à l'ombrage telles que le charme (Carpinus betulus L), le tilleul (Tilia cordata Mill) ou le hêtre (Fagus silvatica L), en donnant la préférence à celle de ces essences dont la compétitivité est la moindre sur la station en question. C'est ainsi que dans le domaine phytosociologique de la hêtraie, on préfère travailler avec le charme, plus docile et amène. L'idée d'implanter le peuplement accessoire en même temps que l'essence principale repose sur la constatation qu'il est préférable 
de contenir par des interventions culturales (recépage) la vigueur de l'essence commensale, plutôt que de devoir l'introduire à grands frais plus tard, d'autant que dans les conditions actuelles de densité de gibier cervidé, il faudrait clôturer la parcelle une seconde fois au moment de l'introduction du peuplement accessoire.

À l'état de fourré, on réglera la densité du massif afin de garantir la survie en quantité suffisante du peuplement accessoire. En revanche, dès l'état de perchis interviennent des éclaircies sélectives vigoureuses qui permettent de garantir un bon développement des essences commensales, qui socialement subordonnées et de stature très svelte en raison de la pénombre, risquent d'être courbées par les neiges précoces, voire même à la suite d'orages estivaux, et de perdre ainsi leurs facultés d'emballage.

Comme le présente la figure 4, avec le début des interventions d'éclaircie sélective, vers l'âge de 30 ans, la surface terrière originellement de 22 à $24 \mathrm{~m}^{2}$ en massifs complets, diminue jusqu'à un niveau de l'ordre de 14 à $16 \mathrm{~m}^{2} / \mathrm{ha}$, pour augmenter progressivement par la suite. Le cours de la surface terrière est donc modulé en fonction des nécessités de sélection. Dès que le peuplement accessoire est suffi-

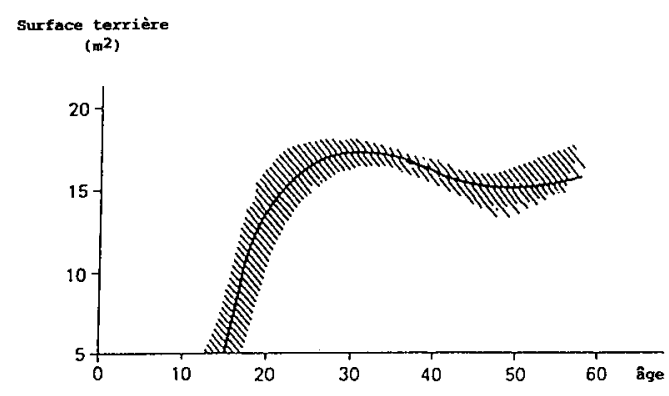

Fig 4. Évolution typique de la surface terrière de jeunes chênaies, traitées selon le concept suisse d'intervention en éclaircies sélectives précoces (d'après Schutz et Badoux, 1979). samment développé pour remplir sa fonction d'emballage, les interventions d'éclaircie se laissent mieux doser, car on intervient parmi les éléments du peuplement accessoire, quand celui-ci est bien fourni, qui accèdent trop rapidement dans l'étage des houppiers et qui exercent une trop grande concurrence, mais dont le départ ne provoque guère d'interruption du couvert. Avec un traitement en éclaircies sélectives vigoureuses, on peut escompter conduire le peuplement à maturité à l'âge d'environ 160 ans.

\section{À PROPOS DE L'ÉDUCATION DU CHÊNE À L'OMBRE EN PETITES TROUÉES}

Dans les ouvrages classiques de sylviculture on convient en raison du tempérament particulièrement héliophile des chênes qu'il faut éduquer cette essence en parcelles suffisamment étendues, d'une surface d'au moins $1 / 2$ ha et que cela nécessite une conduite assez rapide des opérations de rajeunissement. Dans les zones à risques de gels tardifs, la régénération se pratique de préférence et jusqu'à l'état de fourré sous un écran protecteur (d'aulnes par exemple) préalablement introduit (Leibundgut, 1965). On assiste depuis quelques années à un regain d'intérêt pour les techniques de régénération en petites surfaces, voire sans interruption du couvert. II apparaît cependant, au vu des expériences anciennes de régénération notamment en futaie claire, dans des conditions de couvert proches de celles pratiquées en régime de taillis sous futaie, que le succès de telles techniques sylvicoles est loin d'être évident, au contraire. L'expérience montre que sur les bonnes stations le chêne demande d'être rapidement libéré, en trouées d'étendue de l'ordre du bouquet (Perrin, 1954). Cela vaut particulièrement pour le chêne pédonculé, nettement moins tolé- 
rant à l'ombrage que le rouvre. Dans les conditions du Plateau suisse, il n'apparaît pas raisonnable, en raison des problèmes de stabilité envers les neiges lourdes, de régénérer le chêne dans des trouées trop petites, inférieures à 20 à 30 ares (Schütz, 1991, 1992).

\section{RÉFÉRENCES}

Burger $H$ (1921) Studien über die Anzucht und die Massen und Gelderträge der Eiche in der Schweiz. 1. Mitteilung: Ueber morphologische und biologische Eingenschatten der Stiel- und Traubeneiche und ihre Erziehung in Forstgarten. Mitt Schweiz Centralanst Forstl Versuchswes 11, 3, 306-377

Burger $H$ (1944) Ueber die künstliche Begründung von Eichenbeständen. Mitt Schweiz Anst Forstl Versuchswes 23, 2, 283-373

Burger H (1949) Einfluss der Herkunft des Samens auf die Eigenschaften der forstlichen Holzgewächse, VII. Mitteilung: die Eiche. Mitt Schweiz Anst Versuchswes 26, 1, 59-90

Butin H, Kowalski T (1983) Die natürliche Astreinigung und ihre biologischen Voraussetzungen; II. Die natürliche Pilzflora der Stieleiche (Quercus robur L). Eur J For Path 13, 428439

Ellenberg H (1974) Zeigerwerte der Gefässpflanzen Mitteleuropas. Scr Geobot 9, $97 \mathrm{p}$

Kissling $P$ (1983) Les chênaies du Jura central suisse. Mitt Eidg Anst Forstl Versuchswes $59,3,215-401$

Kodrik $J(1991)$ Einfluss des bestandesbildenden Kennziffern auf die Intensität der Beschädigung von Buchenbeständen durch Schnee. In: 3. IUFRO Buchen-Symposium, 3-6 Juni 1988 in Zvolen, 327-330

Krahl-Urban J (1953) Baumtypen bei Eichen und Buchen. Allg Forstz 8, 20, 245-248

Krahl-Urban J (1959) Die Eichen; Forstliche Monographie. Parey, Hamburg \& Berlin

Leibundgut $H$ (1965) Ergebnisse eines Eichenanbauversuches auf dem Hönggerberg. Schweiz Z Forstwes 116, 11/12, 825-833

Leibundgut H (1976) Grundlagen zur Waldpflege: Ergebnisse zwanzigjähriger Untersu- chungen über die Vorgänge der Ausscheidung, Umsetzung und Qualitätsentwicklung in jungen Eichenbeständen. Mitt Eidg Anst Forstl Versuchswes 52, 4, 313-371

Lorne $R$ (1959) Étude quantitative sur les éclaircies dans les peuplements de chêne de qualité. Rev For Fr 11, 746-768

Mayer R (1960) Ueber den wirklichen Wuchsablauf süddeutscher Traubeneichenbestände. Mitt Staatsforstverw Bayerns 31, 137-144

Mitscherlich G (1953) Der Eichenbestand mit Buchen- und Tannenunterstand. Schriftenr Bad Forstl VersAnst, Freiburg i Br 9, 1, 3-35

Pardé J (1962) Table de production pour les forêts de qualité tranchage du secteur ligérien. Stat Rech Exp For Nancy, Notes Techn For, No 11

Perrin $H$ (1954) Sylviculture II. Le traitement des forêts, théorie et pratique des techniques sylvicoles. École nat Eaux et For, Nancy

Roussel L (1977/1978) La formation des gourmands. Bull Soc Forest Franche-Comté 38, $1,1-6 ; 5,99-100 ; 39,1,1-7$

Schütz JP (1979) Le chêne est-il devenu l'enfant pauvre de notre sylviculture? Schweiz $Z$ Forstwes 130, 12, 1047-1070

Schütz JP (1991) Lässt sich die Eiche in der Kleinlochstellung erziehen? Ein Beitrag zur Mischung von Lichtbaumarten. Jahrestag Deutsch Verb forstl Forschungsanst, Sekt Ertragsk, 13-15. Mai 1991, in Treis-Karden/ Mosel

Schütz JP (1992) Die waldbaulichen Formen und die Grenzen der Plenterung mit Laubbaumarten Schweiz. $Z$ Forstwes 143, 6, 442469

Schütz JP, Badoux E (1979) Production de jeunes peuplements de chênes en relation avec la station. Mitt Eidg Anst Forstl Versuchswes 55, 1, 5-141

Spiecker H (1991) Zur Steuerung des Dickenwachstums und der Astreinigung von Trauben- und Stieleichen (Quercus petraea (Matt) Liebl und Quercus robur L). SchrReihe Landesforstverw Baden-Württemberg Nr 72

Trifunovic D (1965) Recueil de tables de production pour les peuplements équiennes des essences principales constituant les forêts de la plaine de Srem: chêne pédonculé, frêne, orme, charme, chêne chevelu, peuplier blanc, saule, robinier. Orig Serb-Cr, Inst Sum Drvn Industr Beograd Posebno izdanje 22, Beograd 